\title{
Comprehensive Procedure for Fast and Accurate Coupled Oscillator Network Simulation
}

\author{
Prateek Bhansali, Shweta Srivastava, Xiaolue Lai and Jaijeet Roychowdhury \\ Department of Electrical and Computer Engineering \\ University of Minnesota \\ Email: \{bhansali, shwetas, laixl, jr\}@umn.edu
}

\begin{abstract}
Coupled oscillator networks occur in various domains such as biology, astrophysics and electronics. In this paper, we present a comprehensive procedure for rapid and accurate simulation of large coupled oscillator networks using widely accepted, fully-nonlinear Perturbation Projection Vector (PPV) phase macromodels. We validate our method against full simulation of $20 \times 20$ coupled network of Brusselator biochemical oscillator and obtain computational speedups of 170x over full simulation. Furthermore, we apply the method to study self-organization phenomenon of Brusselator under asymmetric coupling and time period variations.
\end{abstract}

\section{INTRODUCTION}

Networks of coupled oscillators occur in multitude of physical and natural systems. For example, the dynamics of auto catalytic and oscillating biochemical Brusselator reaction is modeled by coupled oscillators [1]. Natural pacemaker of the heart consists of tens of thousands of cells called sinoatrial node. Each pacemaker cell is an oscillator that produces periodic electrical signals. The reliable and periodic pumping of the heart is driven by rhythmic electrical activity of the network of pacemaker cells [2]. The synchronous flashing of fireflies is yet another example of coupled oscillators in the nature [3]. Each firefly produces flash periodically; but, when fireflies gather together, these insects emit a flash upon the sight of lights produced by neighbouring fireflies. This collaborative behaviour results in a spectacular synchronized flashing. Recently, utility power grid has been studied using a network of coupled oscillators [4]. Each individual oscillator is mathematically modeled by a set of nonlinear, coupled partial differential equations (PDEs). Solving such PDEs analytically, even for a single oscillator, is almost always a formidable task. Thus, analysis and simulation of coupled oscillator network is of great practical importance.

The direct technique is to solve PDEs that governs the dynamics of the oscillator in the time domain. This time-course integration method is not suitable for simulation of oscillators as it inherently accumulates phase errors without limit during simulation [5]. So, hundreds of timesteps per oscillation cycle are required for acceptable accuracy (see Fig. 7), which leads to high computational cost. The situation becomes even worse for coupled oscillator systems with numerous oscillators. Another technique is to use phase domain models, which was pioneered by Winfree [6] and later simplified by Kuramoto [7]. Winfree constructed the model for nearly identical oscillators with the assumption that the coupling among the oscillators is small. This assumption allow the separation of phase and amplitude variations of the oscillator. The amplitude variations die quickly and oscillator relaxes to a steady state limit cycle, since the perturbation from other oscillators is small. Thus, only phase variations need to be tracked to determine the approximate state of the oscillator. In his model, the rate of change of the phase of an oscillator is determined by oscillator's interaction function called "phase sensitivity". However, no algorithm was proposed to obtain these interaction functions. Kuramoto later simplified Winfree's model by approximating the interaction functions as sinusoids. But these sinusoidal interaction functions accumulate significant errors for non-harmonic oscillators
[8], [9]. However, nonlinear PPV macromodel, already established for individual oscillator overcome these errors. Moreover, they can be algorithmically extracted in an computationally efficient manner [5], [10]. The simulation based on PPV macromodel show large simulation speedups due to system size reduction and use of larger timesteps, without affecting accuracy.

In this paper, we provide the procedure and validation for a fast method developed for simulating large networks of coupled oscillators [9]. We start from a PDE description of a specific reactiondiffusion system, the Brusselator, and show how such oscillatory PDE systems can be discretized in space to obtain a network of oscillators coupled spatially. We indicate how perturbation terms required for the application of PPV method can be obtained. This method can be generalized for any spatially coupled electronic or biological oscillators. Finally, using the procedure, we simulate a system of a 200x200 coupled oscillator network under parametric variations.

The remainder of paper is organized as follows. In Section II, a brief review of the nonlinear PPV phase macromodel is presented. Section III deals with Brusselator biochemical oscillator model description and details to develop differential-algebraic equation (DAE) of the oscillator. We show how the PPV phase macromodel of an independent oscillator can be abstracted from the PDE of the Brusselator by observing perturbation terms. In Section IV, details to solve the oscillator network for phase of each oscillator and using them to reconstruct the oscillator states across the network are presented. In Section V, we validate our method against a full simulation for a coupled oscillator network, and show phase error accumulation in time-course transient simulation of a simple two oscillator network. Next, in Section VI, collective behaviour of the Brusselator system is studied under asymmetric coupling strength and time period variations.

\section{NONLINEAR PHASE MACROMOdeL}

In this section, we review the nonlinear phase macromodelling technique using the PPV phase macromodel [5]. The PPV phase macromodels are well suited for large systems involving coupled oscillators as they dramatically improves efficiency and accuracy. A starting point is the differential equation which describes oscillator under external perturbations

$$
\frac{d \vec{x}}{d t}+\vec{f}(\vec{x})=\vec{b}(t)
$$

where $\vec{x}(t)$ is a vector of oscillator states and $\vec{b}(t)$ represents the vector of perturbation signals applied to corresponding states of free running oscillator. Assuming $\vec{b}(t)$ and the amplitude variations are small, the solution to this oscillator subject to perturbation can be shown [5] to take the following form

$$
\vec{x}_{p}(t)=\vec{x}_{S}(t+\alpha(t))
$$

where $\vec{x}_{p}(t)$ represents the waveform of the perturbed oscillator, $\vec{x}_{S}(t)$ is the steady state periodic solution of unperturbed oscillator and $\alpha(t)$ is the phase deviation caused by the external perturbation $\vec{b}(t)$. In [5], Demir et al proved that the phase deviation $\alpha(t)$ is governed by a 
simple scalar equation

$$
\frac{d \alpha(t)}{d t}=\vec{V}_{1}^{T}(t+\alpha(t)) \cdot \vec{b}(t)
$$

where $\vec{V}_{1}^{T}$ is the PPV of system size. Each component in PPV represents the oscillator's phase sensitivity to the applied perturbation signal. In the next section, we apply PPV macromodelling technique to single Brusselator biochemical oscillator and show how this technique of the phase macromodelling can be extended to coupled oscillator networks. The PPV macromodels can be obtained both in frequency and time domain algorithmically [10], unlike Winfree's interaction functions.

\section{DESCRIPTION OF THE NUMERICAL PROCEDURE FOR BRUSSELATOR EQUATION}

It is well known that many types of biochemical reaction-diffusion (RD) systems [1] produce intricate and beautiful spatial patterns ("phase waves"). For example, the patterns in Fig. 11(b) result from a simple type of biochemical RD system called the Brusselator.

The PDEs of a RD system have the form

$$
\frac{\partial \vec{c}(x, y, t)}{\partial t}=R(\vec{c}, t)+D_{c} \nabla^{2} \vec{c}
$$

where $x, y$ are spatial dimensions and $\vec{c}$ is a vector of concentrations of the chemical species taking part in RD reaction. The generation speed of chemical species is given by $R(\vec{c}, t)$ and its functional form depends on the specific system being studied. The diffusion coefficients are represented by matrix $D_{c}$ and second term in the RHS of (4), $D_{c} \nabla^{2} \vec{c}$, represents change in the $\vec{c}$ by diffusion. For example, the Brusselator system is modeled by the following nonlinearly coupled PDEs [11]

$$
\begin{aligned}
& \frac{\partial u}{\partial t}=A-(B+1) u+u^{2} v+D_{u} \nabla^{2} u \\
& \frac{\partial v}{\partial t}=B u-u^{2} v+D_{v} \nabla^{2} v
\end{aligned}
$$

where $A$ and $B$ are constants specific to reaction, $u$ and $v$ are the chemical concentrations of two species, and their temporal derivatives represent the rate of change of their concentrations with time. The diffusion coefficients are represented as $D_{u}$ and $D_{v}$. We use (5) to present the coupled oscillator simulation method.

In order to solve (5) numerically, we choose rectangular grid as shown in Fig. 1 to discretize PDEs spatially. Let the grid points be denoted by $(i, j)$, where $i \in\left\{1, N_{1}\right\}, j \in\left\{1, N_{2}\right\}$ and thus the network size is $N_{1} \times N_{2}$. Each grid point represents an oscillator, as we will verify later, described by the nonlinearly coupled PDE (5). The line joining the grid points corresponds to coupling of an oscillator with its neighbour.

To discretize the PDE (5), we use second-order central difference scheme for the Laplacian operator, $\nabla^{2}$, obtained using Taylor series expansion as

$$
\begin{aligned}
\left.\nabla^{2} u\right|_{i, j} \simeq & \frac{u_{i-1, j}(t)+u_{i+1, j}(t)-2 u_{i, j}(t)}{\Delta x^{2}} \\
& +\frac{u_{i, j-1}(t)+u_{i, j+1}(t)-2 u_{i, j}(t)}{\Delta y^{2}} \\
\left.\nabla^{2} v\right|_{i, j} \simeq & \frac{v_{i-1, j}(t)+v_{i+1, j}(t)-2 v_{i, j}(t)}{\Delta x^{2}} \\
& +\frac{v_{i, j-1}(t)+v_{i, j+1}(t)-2 v_{i, j}(t)}{\Delta y^{2}}
\end{aligned}
$$

where $\Delta x$ and $\Delta y$ are space discretization steps in $x$ and $y$ dimensions respectively. For boundary points, we use non-flux boundary

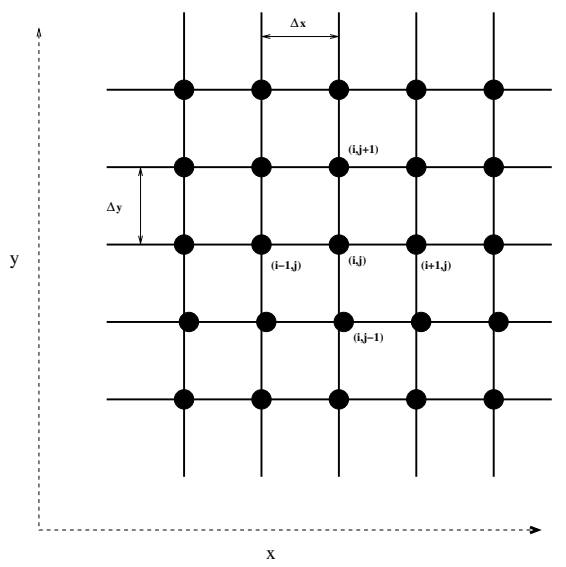

Fig. 1. Rectangular grid for discretizing the Brusselator PDEs.

conditions given as

$$
\begin{aligned}
& \left(\frac{\partial u}{\partial n}\right)_{i=\left[1, N_{1}\right] ; j=\left[1, N_{2}\right]}=0 \\
& \left(\frac{\partial v}{\partial n}\right)_{i=\left[1, N_{1}\right] ; j=\left[1, N_{2}\right]}=0
\end{aligned}
$$

where $\mathrm{n}$ is normal to the boundary. The boundary conditions play a direct role in determining the perturbations across the grid. Then, (5) is evaluated at each grid point using (6) and (7). For example, at any internal point $(i, j)$ we have

$$
\begin{aligned}
\frac{d}{d t} u_{i, j}(t) & =A-(B+1) u_{i, j}(t)+u_{i, j}^{2}(t) v_{i, j}(t)+\left(C_{u}(t)\right)_{i, j} \\
\frac{d}{d t} v_{i, j}(t) & =B u_{i, j}(t)-u_{i, j}^{2}(t) v_{i, j}(t)+\left(C_{v}(t)\right)_{i, j}
\end{aligned}
$$

where,

$$
\begin{aligned}
\left(C_{u}(t)\right)_{i, j}=D_{u} & {\left[\frac{u_{i-1, j}(t)+u_{i+1, j}(t)-2 u_{i, j}(t)}{\Delta x^{2}}\right.} \\
+ & \left.\frac{u_{i, j-1}(t)+u_{i, j+1}(t)-2 u_{i, j}(t)}{\Delta y^{2}}\right], \\
\left(C_{v}(t)\right)_{i, j}=D_{v} & {\left[\frac{v_{i-1, j}(t)+v_{i+1, j}(t)-2 v_{i, j}(t)}{\Delta x^{2}}\right.} \\
& \left.+\frac{v_{i, j-1}(t)+v_{i, j+1}(t)-2 v_{i, j}(t)}{\Delta y^{2}}\right] .
\end{aligned}
$$

For simplicity, we define $c_{u x}, c_{u y}, c_{v x}$ and $c_{v y}$ as

$$
\begin{gathered}
c_{u x}=\left(\frac{D_{u}}{\Delta x^{2}}\right), c_{u y}=\left(\frac{D_{u}}{\Delta y^{2}}\right), \\
c_{v x}=\left(\frac{D_{v}}{\Delta x^{2}}\right), c_{v y}=\left(\frac{D_{v}}{\Delta y^{2}}\right) .
\end{gathered}
$$

The diffusion terms, $C_{u}(t)$ and $C_{v}(t)$, contribute to perturbations of an oscillator from other oscillators in the grid. Thus, the characteristics of a single oscillator can be captured by eliminating the diffusion terms, $C_{u}(t)$ and $C_{v}(t)$, from (8) as

$$
\begin{aligned}
\frac{d}{d t} u(t)-A+(B+1) u(t)-u^{2}(t) v(t) & =0 \\
\frac{d}{d t} v(t)-B u(t)+u^{2}(t) v(t) & =0
\end{aligned}
$$

Note that in the above equation, we have dropped the grid point index $(i, j)$ to make it clear that if we put the diffusion terms equal to zero, 
there will be no coupling effect or perturbations from the neighboring oscillators and the PDE at each node in the network will be the same and can be represented by (11).

Next we develop the DAE of (11). It will be same for every oscillator in the grid as we have eliminated the diffusion terms, $C_{u}(t)$ and $C_{v}(t)$. These terms will be incorporated later while solving for the coupled oscillator network (in Section IV) as external perturbations to an oscillator. To represent the PDEs (11) of the Brusselator in a convenient circuit's DAE form, we start with

$$
\frac{d}{d t} \vec{q}(\vec{x})+\vec{f}(\vec{x})+\vec{b}(t)=\overrightarrow{0}
$$

where $\vec{x}$ are the unknowns. For (11) we can write, $\vec{q}(\vec{x})=\vec{x}$ and $\vec{b}(t)$ is the vector of perturbations from neighbouring oscillators. Thus, for an independent oscillator, $\vec{b}(t)=0$. Next, we consider $\vec{x}=[u(t) v(t)]^{T}$, then $\vec{f}(\vec{x}), \frac{\partial \vec{f}}{\partial \vec{x}}$ and $\frac{\partial \vec{q}}{\partial \vec{x}}$ for (11) can be written as

$$
\begin{aligned}
\vec{x} & =\left(\begin{array}{l}
u(t) \\
v(t)
\end{array}\right) \\
\vec{f}(\vec{x}) & =\left(\begin{array}{cr}
-A+(B+1) u(t)-u^{2}(t) v(t) \\
-B u(t)+u^{2}(t) v(t)
\end{array}\right) \\
\frac{\partial \vec{f}}{\partial \vec{x}} & =\left(\begin{array}{cr}
B+1-2 u(t) v(t) & -u^{2}(t) \\
-B+2 u(t) v(t) & u^{2}(t)
\end{array}\right) \\
\frac{\partial \vec{q}}{\partial \vec{x}} & =\left(\begin{array}{ll}
1 & 0 \\
0 & 1
\end{array}\right)
\end{aligned}
$$

Using the above DAE of the Brusselator, we extract the steady state waveform and PPV [10] of $u$ and $v$ for $A=0.5, B=1.7$ using the harmonic balance method. The waveforms obtained are shown in Fig. 2(a) and Fig. 2(b).

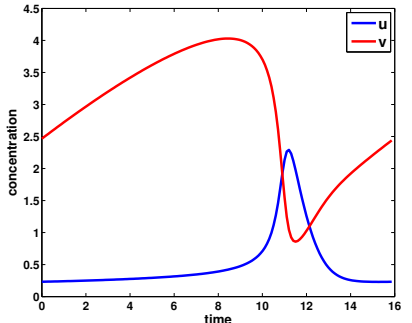

(a) Plot of steady state.

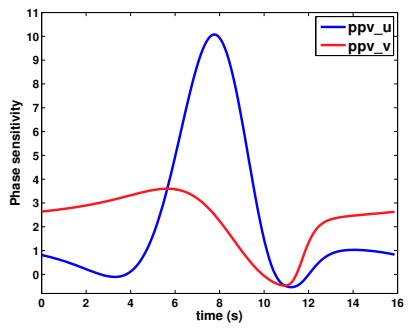

(b) Plot of PPVs.
Fig. 2. Plot of the steady state and the PPV of $u$ and $v$.

The PPV waveforms need to be extracted only once and can be reused for a coupled oscillator network simulation as we show next.

\section{Simulation Details of Coupled Oscillator Network USING PPV MACROMODELS}

In this section, we present the details for simulating large coupled oscillator networks. The PPV phase macromodelling technique for a single oscillator can be extended for coupled oscillator networks, by modeling $\vec{b}(t)$ in (3) to account for coupling among oscillators. For example, the oscillators at grid points $(i, j+1),(i, j-1),(i+1, j)$ and $(i-1, j)$ cause perturbations in oscillator states at grid point $(i, j)$ through coupling as shown in Fig. 3. The vector of perturbations, $\vec{b}_{i, j}(t)$, at the grid point $(i, j)$ can be obtained by comparing (8) and (11). The components of $\vec{b}_{i, j}(t)$ are $\left(C_{u}(t)\right)_{i, j}$ and $\left(C_{v}(t)\right)_{i, j}$, for the $u$ and $v$ state of the oscillator respectively. Therefore, for any grid point $(i, j)$ in the network, perturbation vector $\vec{b}_{i, j}(t)$ can be written

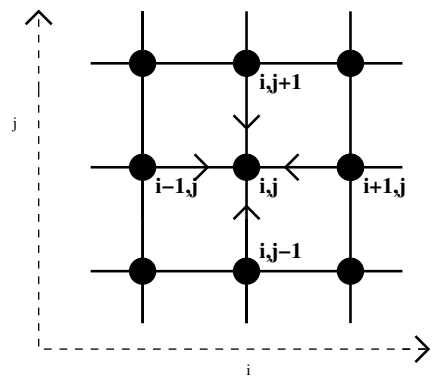

Fig. 3. Contribution of perturbation from the neighboring grid points.

as

$$
\vec{b}_{i, j}(t)=\left(\begin{array}{l}
b \_u, u_{i, j}(t) \\
b_{-} v_{i, j}(t)
\end{array}\right)
$$

where,

$$
\begin{aligned}
& b \_u_{i, j}(t)=\left(C_{u}(t)\right)_{i, j} \\
& b \_v_{i, j}(t)=\left(C_{v}(t)\right)_{i, j}
\end{aligned}
$$

Next, we solve for the phase deviation of oscillators in the network using PPV, $\vec{V}_{1}^{T}(t)$ and the perturbation signal, $\vec{b}(t)$.

At any point $(i, j)$, the phase deviation $\alpha_{i, j}(t)$ can be calculated using (3) as the solution of following equation

$$
\begin{aligned}
\dot{\alpha}_{i, j}(t) & =\vec{V}_{1}^{T}\left(t+\alpha_{i, j}(t)\right) \cdot \vec{b}_{i, j}(t) \\
\dot{\alpha}_{i, j}(t) & =\left(p p v \_u\left(t+\alpha \alpha_{i, j}(t)\right) \quad p p v \_v\left(t+\alpha_{i, j}(t)\right)\right) \cdot\left(\begin{array}{l}
b \_u_{i, j}(t) \\
b \_v_{i, j}(t)
\end{array}\right) \\
& =p p v \_u\left(t+\alpha_{i, j}(t)\right) b \_u_{i, j}(t)+p p v \_v\left(t+\alpha_{i, j}(t)\right) b \_v_{i, j}(t) \\
& =f\left(t, \alpha_{i, j}(t)\right)
\end{aligned}
$$

where $p p v \_u(t)$ and $p p v \_v(t)$ are PPVs of for $u$ and $v$, respectively.

The second order Runge-Kutta (RK2) numerical method can be used to solve for $\vec{\alpha}(t)$. According to RK2 algorithm, the phase deviation for point $(i, j)$ at time $t_{n+1}=t_{n}+h$, where $h$ is timestep used in the simulation, is solved as shown

$$
\begin{aligned}
\left.\alpha_{n+1}\right|_{i, j}= & \left.\alpha_{n}\right|_{i, j}+\left.k_{2}\right|_{i, j} \\
\left.k_{2}\right|_{i, j}= & h f\left(t_{n}+\frac{h}{2},\left.\alpha_{n}\right|_{i, j}+\frac{\left.k_{1}\right|_{i, j}}{2}\right) \\
= & h\left[p p v \_u\left(t_{n}+\frac{h}{2}+\left.\alpha_{n}\right|_{i, j}+\frac{\left.k_{1}\right|_{i, j}}{2}\right) b \_u_{i, j}\left(t_{n}+\frac{h}{2}\right)\right. \\
& \left.+p p v \_v\left(t_{n}+\frac{h}{2}+\left.\alpha_{n}\right|_{i, j}+\frac{\left.k_{1}\right|_{i, j}}{2}\right) b_{-} v_{i, j}\left(t_{n}+\frac{h}{2}\right)\right]
\end{aligned}
$$

and $k_{1}$ is calculated as

$$
\begin{aligned}
\left.k_{1}\right|_{i, j}= & h f\left(t_{n},\left.\alpha_{n}\right|_{i, j}\right) \\
= & h\left[p p v \_u\left(t_{n}+\left.\alpha_{n}\right|_{i, j}\right) b \_u_{i, j}\left(t_{n}\right)\right. \\
& \left.+p p v \_v\left(t_{n}+\left.\alpha_{n}\right|_{i, j}\right) b \_v_{i, j}\left(t_{n}\right)\right]
\end{aligned}
$$

Assuming the amplitude variations are small, the perturbed solutions for $u(t)$ and $v(t)$ under the effect of $\vec{b}(t)$ are given by (2). Thus, for grid point $(i, j)$ at time $t=t_{n}$

$$
\begin{aligned}
& u_{i, j}\left(t_{n}\right)=u_{s s}\left(t_{n}+\alpha_{i, j}(t)\right) \\
& v_{i, j}\left(t_{n}\right)=v_{s s}\left(t_{n}+\alpha_{i, j}(t)\right)
\end{aligned}
$$


where $u_{s s}\left(t_{n}\right)$ and $v_{s s}\left(t_{n}\right)$ are the steady state solution at $t=t_{n}$ in the absence of the perturbation signal, $\vec{b}(t)$.

Using the above procedure, we calculate the phase deviation, $\vec{\alpha}(t)$ and use it to compute the perturbed solution, $\vec{u}(t)$ and $\vec{v}(t)$ of the network.

\section{NuMERICAL RESUlts}

In this section, we apply and validate the technique presented above using the Brusselator oscillator. A simple, coupled oscillator network of two oscillators is represented schematically in Fig. 4. The line joining the oscillators represent the coupling between $u$ and $v$ nodes of each oscillator. We first simulate the two oscillator network using the PPV phase macromodelling technique for coupled oscillators. The two oscillators start with random initial phase between 0 and $2 \pi$ and we take $A=0.5, B=1.7$ and $c_{u x}=c_{u y}=c_{v x}=c_{v y}=0.025$.

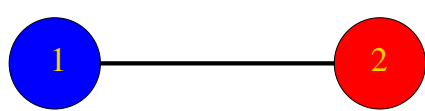

Fig. 4. Schematic of two coupled oscillators.

The phase deviation and oscillator state waveforms obtained are shown in Fig. 5 and Fig. 6. After a few oscillation cycles, $\frac{d \vec{\alpha}}{d t}$ becomes constant, indicating that the oscillators get phase locked. We can see from $u$ waveforms in Fig. 6 that oscillators indeed get phase locked. The coupling between the two oscillators forces them to get into phase.

Now, we compare the results obtained with the time-course full simulation of the above system, for different time steps under same initial conditions. The full simulation results are computed using same system parameters and RK2 numerical method. From Fig. 7, we can see the efficiency of proposed method against the full simulation. For example, using 100 timesteps per oscillation cycle in the full simulation and the proposed method, the former method accumulates significant phase errors. However, when we increase the number of timesteps in the full simulation the phase error reduces. As it can be seen, the result closely match with larger number of time points in the full simulation.

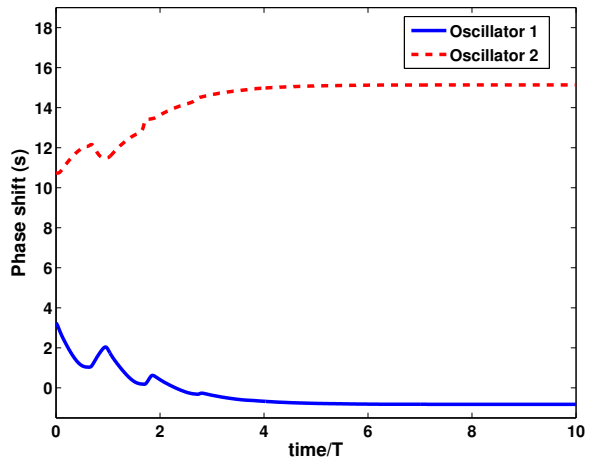

Fig. 5. Phase shift in the two oscillator using the nonlinear PPV phase macromodel.

Next, we simulate a 20x20 coupled Brusselator oscillator network using full system simulation and proposed method for similar initial conditions and a non-flux boundary condition for boundary points. The parameters $A, B, c_{u x}, c_{u y}, c_{v x}$ and $c_{v y}$ are chosen to be the same as before. We take 200 timesteps per oscillation cycle for PPV based simulation and 1500 timesteps for full simulation, to get comparable accuracies. We plot the simulation results of $u$ state of oscillator at position $(1,1)$ and $v$ state of oscillator at $(10,10)$ in the coupled network in Fig. 8 and Fig. 9. It can be seen from Fig. 8 and Fig. 9 that nonlinear PPV based coupled oscillator simulation method results in a close match with full system simulation (with sufficiently high number of time steps), which validates our method. However, the proposed method is computationally cheaper than the full simulation.

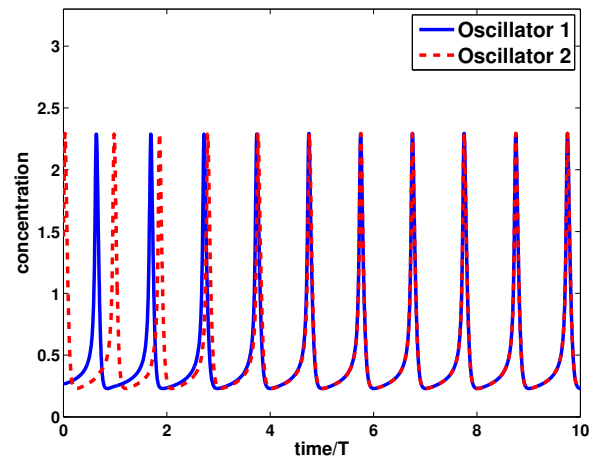

Fig. 6. Phase locked $u$ waveforms of two oscillators using PPV macromodel.

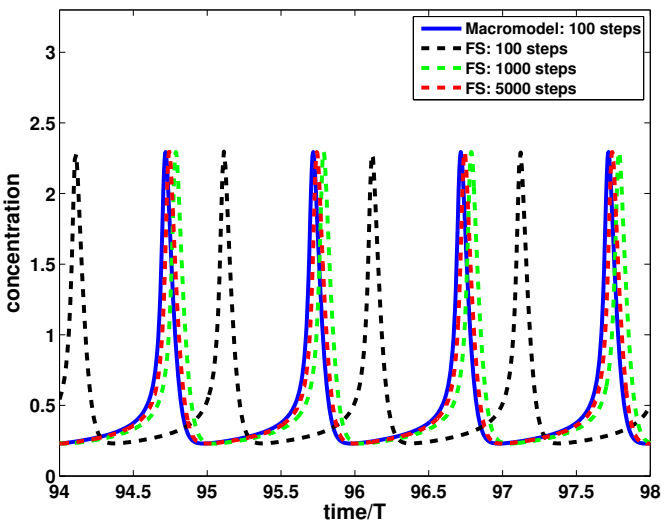

Fig. 7. Plot of $u$ waveform for oscillator 1 using PPV macromodel and full simulation (FS).

On an AMD Athlon64 3800+ based workstation, running MATLAB on Linux kernel 2.61.19, our technique using PPV phase macromodels took $70 \mathrm{~s}$ for 100 oscillation cycles as compared to $12000 \mathrm{~s}$ with full simulation for comparable accuracy (implemented on the same workstation). This is a 170-fold speedup and is expected to go higher for large coupled oscillator networks, involving a larger number of oscillators. The speed up is due to the fact that we calculate nonlinear phase macromodel only once and use that across the network for further analysis.

\section{EXPLORING SELF-ORGANIZATION UNDER ASYMMETRIC COUPLING AND TIME PERIOD VARIATION}

In this section, we simulate a larger coupled oscillator network, using the procedure developed in the previous sections to explore the effect of asymmetric coupling and time period variations in the network for random and given initial conditions of $\vec{\alpha}$ at $t=0$. The Brusselator self-organizes itself forming spatio-temporal patterns and this has been experimentally observed [1], [12]. The network is made up of $200 \times 200$ identical oscillators, with free running frequency of each oscillator as $f_{0}=T_{0}{ }^{-1}$ and coupling parameters $c_{u x}, c_{u y}, c_{v x}$ and $c_{v y}$, as defined in Section III. The self-organization and subsequent pattern formation depends on $A, B$ and coupling parameters. For simulation purposes, we chose $A=0.5, B=1.7$ and nominal $c_{u x}{ }^{-1}=40, c_{u x}{ }^{-1}=40, c_{u x}{ }^{-1}=40$ and $c_{u x}{ }^{-1}=40$. 


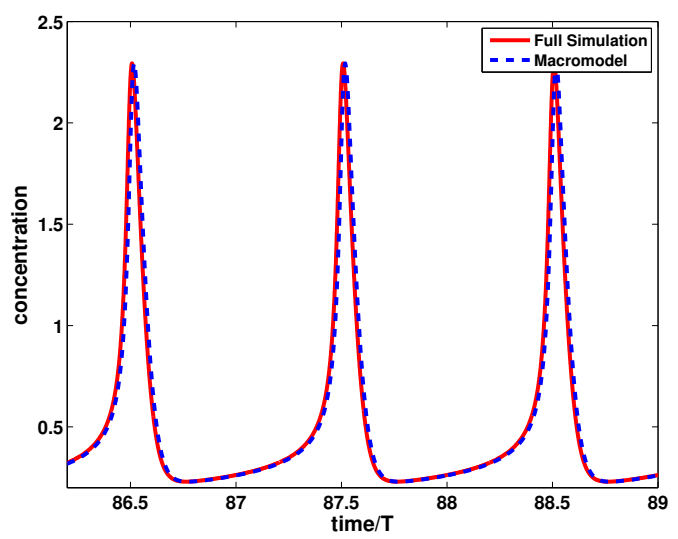

Fig. 8. Plot of $u$ waveform of $(1,1)$ oscillator.

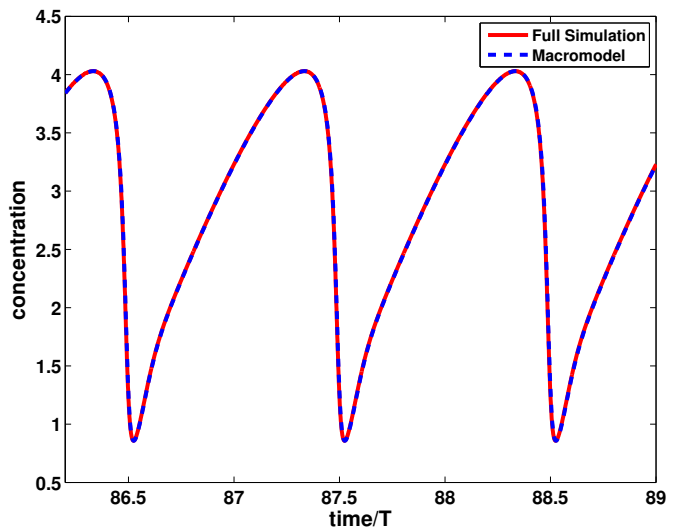

Fig. 9. Plot of $v$ waveform of $(10,10)$ oscillator.

\section{A. Random initial condition simulations}

The oscillators in the grid are assigned random initial phase between 0 to $2 \pi$ as shown in Fig. 10. Each small dot in the phase plot represents the phase of that oscillator. The oscillators with the same phase have the same color. The network is simulated for 200 oscillation cycles $(\mathrm{t}=200 \mathrm{~T})$ and solved for $\vec{\alpha}(t)$.

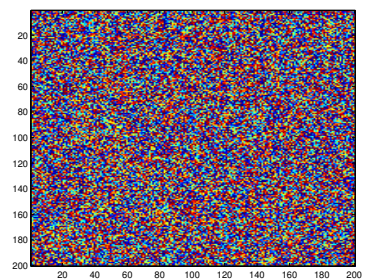

Fig. 10. Random initial phase of oscillators in the network at $\mathrm{t}=0$.

1) Network simulation without variation: We simulate the network with random initial phase for each oscillator. For simulation without any variation in the coupling parameter or the time period, Fig. 11 shows the phase plot of oscillators at different time instants. As time evolves, the mutual coupling between the oscillators forces phase lock between them in a localized fashion, which can be seen in the form of spiral patterns or phase waves in Fig. 11(a) at $t=50 T$. These patterns grow and emerges as seen in Fig. 11(b) at $\mathrm{t}=200 \mathrm{~T}$. We call these spirals as seed.

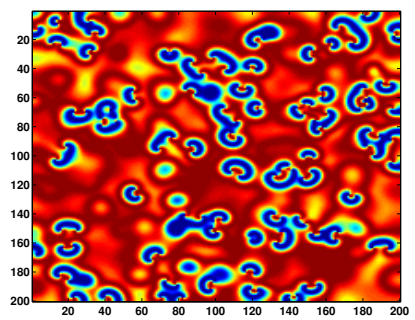

(a) Phase plot at $\mathrm{t}=50 \mathrm{~T}$.

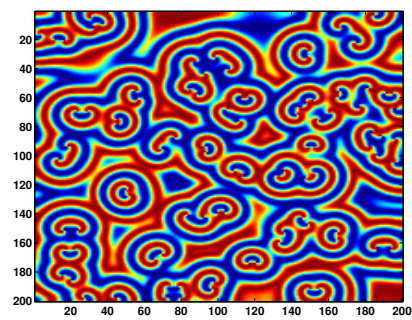

(b) Phase plot at $\mathrm{t}=200 \mathrm{~T}$.
Fig. 11. Phase plot of the network with random initial conditions.

2) Network simulation with variations: We now simulate the network with small variations in the time period, $T$ and in coupling parameters. In the first simulation, the coupling related parameters $c_{u x}{ }^{-1}, c_{u y}{ }^{-1}, c_{v x}{ }^{-1}$ and $c_{v y}{ }^{-1}$ are varied is such a way that they follow a Gaussian distribution, N(40,49). The plot of the phase of oscillators at $t=200 T$ is shown in Fig. 12. It can be seen that spiral patterns, almost similar to Fig. 11, are formed and network is robust against variations in coupling. This variations can be physically mapped to the anisotropy of the physical system under consideration. For example, heart is not isotropic and homogeneous in all directions [13]. A practical and useful application of the proposed method can be to simulate auto-oscillatory cells of the mammalian heart.

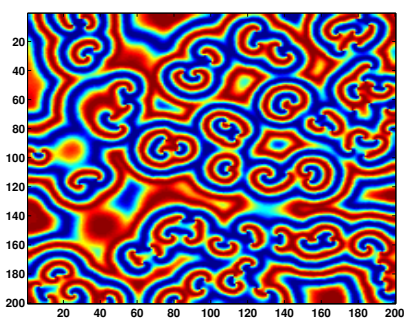

(a) Phase plot at $\mathrm{t}=200 \mathrm{~T}$.

Fig. 12. Phase plot with random initial phase and variations in the coupling parameters.

In the second simulation, the normalized time period of oscillators in the network is varied as a Gaussian distribution, $\mathrm{N}(1,1 \mathrm{e}-7)$. The phase plots are shown in Fig. 13. Again, similar patterns form with small random time-period variations, but the pattern formation in the network is not robust against it.

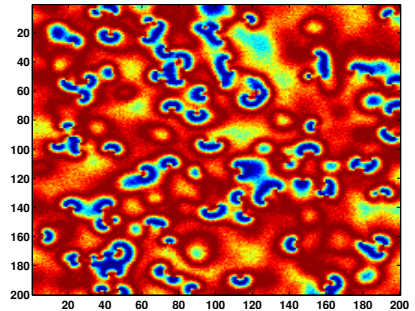

(a) Phase plot at $\mathrm{t}=50 \mathrm{~T}$.

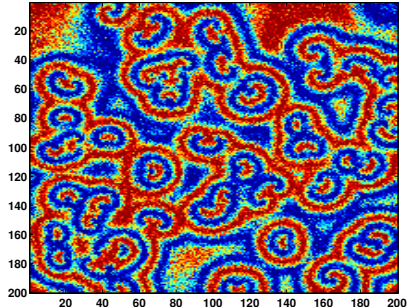

(b) Phase plot at $\mathrm{t}=200 \mathrm{~T}$.
Fig. 13. Phase plot with random initial phase and variations in the time period.

\section{B. Given initial condition simulations}

In this subsection, we analyze the collaborative behaviour of the oscillators in the network for a given initial phase. We start by placing a seed at the center of network as shown in Fig. 14, enclosed by a dashed rectangle for illustration purpose, and analyze its growth. 
Further, we demonstrate the collaborative behaviour of oscillators in the network under parametric variations for given initial conditions.

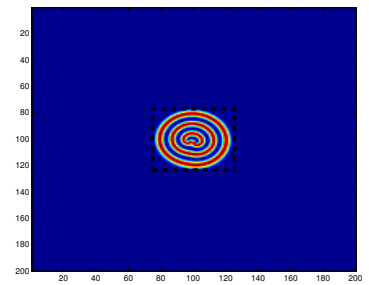

Fig. 14. Phase plot of oscillators with given initial condition (seeded).

1) Simulation without noise: After placing the seed, we simulate the network for similar values of $A, B$ and coupling parameters mentioned in Section VI-A. This results in a stable spiral pattern growth encompassing the whole network and phase synchronization among oscillators takes place, as illustrated in Fig. 15.

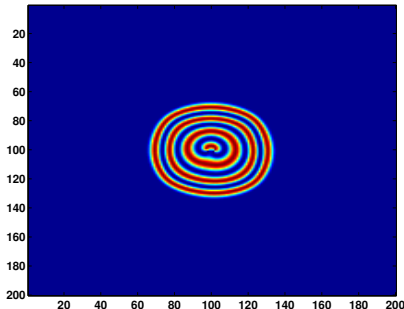

(a) Phase plot at $\mathrm{t}=50 \mathrm{~T}$. (b) Phase plot at $\mathrm{t}=200 \mathrm{~T}$.

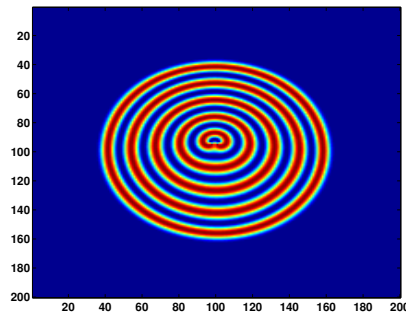

Fig. 15. Phase plot with given initial conditions illustrating seed growth.

2) Simulation with noise: In a similar manner as in Section VI(A), we investigate the effect of parameter variations on the seed growth. In the first simulation, we simulate the network with Gaussian noise in the coupling parameters, $c_{u x}{ }^{-1}, c_{u y}{ }^{-1}, c_{v x}{ }^{-1}$ and $c_{v y}{ }^{-1}$, of $\mathrm{N}(40,49)$ i.e. with a standard deviation (SD) of $17.5 \%$. The phase plot at different time instants is shown in Fig. 16. It can be seen that the network is robust and basic elliptical patterns are still present.

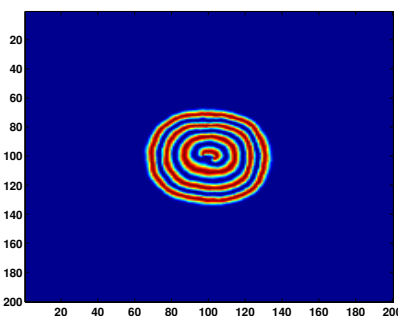

(a) Phase plot at $\mathrm{t}=50 \mathrm{~T}$.

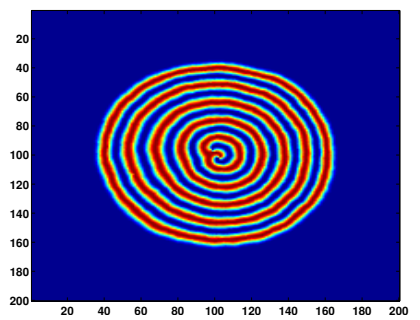

(b) Phase plot at $\mathrm{t}=200 \mathrm{~T}$.
Fig. 16. Phase plot with seeded network and variations in coupling parameter.

In the second simulation, we simulate the network with normalized time period varying as a Gaussian distribution, $\mathrm{N}(1,1 \mathrm{e}-7)$, across the network. The phase plots at different time instants are shown in Fig. 17. Thus, the network is affected by the noise in time period but the basic seed pattern remain the same.

\section{CONCLUSIONS}

We have presented a comprehensive procedure for simulation of coupled oscillator networks using the phase-domain nonlinear PPV macromodels. The method is generally applicable to any spatially coupled oscillator network. We have validated our method for 20x20

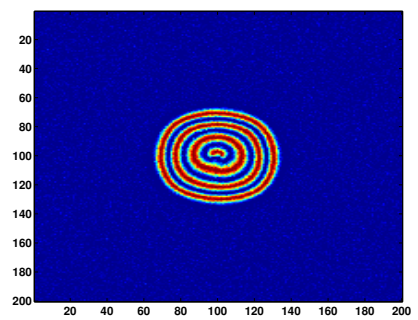

(a) Phase plot at $\mathrm{t}=50 \mathrm{~T}$.

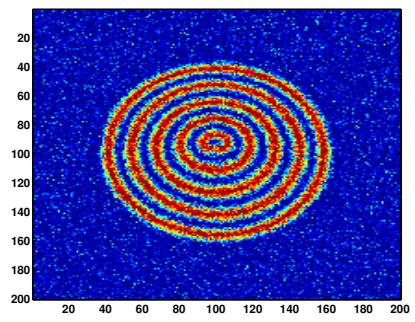

(b) Phase plot at $\mathrm{t}=200 \mathrm{~T}$.
Fig. 17. Phase plot with initial seed and Gaussian noise in time periods.

coupled Brusselator biochemical oscillator demonstrating speedups over the time-course full simulation. In addition, the proposed method provides useful insights into self-organization phenomenon of Brusselator under parametric variations. Currently, we are working on its application to simulate the conduction system of heart.

\section{Acknowledgments}

We thank the MARCO GSRC program, which supported this work. Computational and infrastructural resources from the University of Minnesota's Digital Technology Center is gratefully acknowledged.

\section{REFERENCES}

[1] P. K. Maini, K. J. Paintera, and H. N. P. Chaub. Spatial pattern formation in chemical and biological systems. J. Chem. Soc., Faraday $T$ rans., pages 3601-3610, 1997.

[2] Alexander V. Panfilov and Arun V. Holden. Computational Biology of the Heart. John Wiley and Sons, Chichester, 1997.

[3] Steven Strogatz. Sync: The Emerging Science of Spontaneous Order. Hyperion, 2003.

[4] G. Filatrella, A. H. Nielsen, and N. F. Pedersen. Analysis of a power grid using a kuramoto-like model. The European Physical Journal B Condensed Matter and Complex Systems, 61:485-491, 2008.

[5] A. Demir, A. Mehrotra, and J. Roychowdhury. Phase noise in oscillators: a unifying theory and numerical methods for characterization. IEEE Trans. on Circuits and Systems-I:Fundamental Theory and Applications, 47(5):655-674, May 2000.

[6] A. T. Winfree. Biological rhythms and the behavior of populations of coupled oscillators. J. Theor. Biol., 1967.

[7] Y. Kuramoto. Chemical Oscillations, Waves, and turbulence. Springer, Berlin, 1984

[8] Steven H. Strogatz. From kuromoto to crawford: exploring the onset of synchronization in populations of coupled oscillators. Physica D, 2000.

[9] X. Lai and J. Roychowdhury. Fast simulation of large networks of nanotechnological and biochemical oscillators for investigating selforganization phenomena. In Proc. IEEE Asia South-Pacific Design Automation Conference, January 2006.

[10] A. Demir and J. Roychowdhury. A reliable and efficient procedure for oscillator ppv computation, with phase noise macromodelling applications. IEEE Trans. on Computer-Aided Design of Integrated Circuits and Systems, 22(2):188-197, February 2003.

[11] Irving R Epstein and John A Pojman. An introduction to nonlinear chemical dynamics : oscillations, waves, patterns, and chaos. New York : Oxford University Press, 1998.

[12] A. L. Lin, A. Hagberg, A. Ardelea, M. Bertram, H. L. Swinney, and E. Meron. Four-phase patterns in forced oscillator systems. Physical Review E, 62:3790-3798, 2000.

[13] E. Etienne Verheijck, Andy Wessels, Antoni C. G. van Ginneken, Jan Bourier, Marry W. M. Markman, Jacqueline L. M. Vermeulen, Jacques M. T. de Bakker, Wouter H. Lamers, Tobias Opthof, , and Lennart N. Bouman. Distribution of atrial and nodal cells within the rabbit sinoatrial node : Models of sinoatrial transition. Circulation, 97:1623-1631, 1998. 